Política y Sociedad

ISSN-e: 1988-3129

\title{
La Planificación hidrológica como gobierno compartido o el nexo entre sostenibilidad y federalismo
}

\author{
Alberto De La Peña Varona ${ }^{1}$ y Jaione Mondragón Ruiz de Lezana²
}

Enviado: 08-03-2021 // Aceptado: 04-01-2022

Resumen. La política hidrológica española, especialmente a partir de la aprobación de la Directiva Marco del Agua, se diseña e implanta de acuerdo al paradigma de gobernanza multinivel, al igual que sucede en otros Estados europeos. Particularmente en el caso de las demarcaciones hidrológicas intercomunitarias, ello va a suponer un desafío al modelo territorial del Estado autonómico, al exigir nuevos procedimientos para integrar la diversidad territorial en las decisiones comunes. Esto es, la política de aguas va a demandar nuevas prácticas de gobierno compartido, lo que no es sencillo de encajar en un sistema como el español, en el que frecuentemente se ha destacado un desarrollo relativamente escaso de sus dinámicas de colaboración intergubernamental, tanto por determinadas características institucionales como por una cierta cultura política y percepción territorial poco acordes al federalismo.

El objetivo de este trabajo será, por tanto, explorar y describir las características generales de una dimensión concreta de la política de aguas como es la planificación hidrológica, presentándola como pauta de gobierno compartido a partir de la referencia concreta del caso español y su modelo territorial. Este es un análisis que estimamos va más allá de lo meramente descriptivo, por cuanto concluimos que la proyección espacial de determinadas políticas públicas ligadas a la sostenibilidad ambiental podría inducir a los actores participantes a modificar las maneras tradicionales de entender el territorio, interiorizando valores como la diversidad e interdependencia y, en definitiva, profundizando en el tránsito al federalismo.

Palabras clave: gobernanza del agua; planificación hidrológica; modelo de Estado; sostenibilidad; territorio.

\section{[en] Water planning and shared government or the link between sustainability and federalism}

\begin{abstract}
The Spanish water policy, particularly after the Water Framework Directive, is being designed and implemented according with the multilevel governance paradigm as it is the case in other European countries. Particularly in the inter-regional basins, this new model challenges the so-called autonomous State as it requires new collaborative inter-administrative procedures in order to integrate different regional interests into nation-wide decisions. Water policy is going to demand new practices of shared government, which is not easy to fit into a system like the Spanish one, in which a relatively low development of its dynamics of intergovernmental collaboration has often been highlighted, both due to certain institutional characteristics as for a certain political culture and territorial perception not very consistent with federalism.

The aim of this paper will therefore be to explore and describe the general characteristics of a specific dimension of water policy such as hydrological planning, presenting it as an example of shared governance based on the specific reference of the Spanish case and its territorial model. An analysis that we believe goes beyond the merely descriptive, in that we conclude that the spatial projection of certain public policies linked to environmental sustainability could induce the participating actors to modify the traditional ways of understanding the territory, internalising values such as diversity and interdependence and, in short, deepening the transition to federalism.
\end{abstract}

Keywords: water governance; water planning; territorial state-model; sustainability; territory.

Sumario. 1. Introducción. 2. El Estado de las autonomías. 3. La Directiva Marco del Agua: gobernanza multinivel en el tránsito a la sostenibilidad. 4. La planificación hidrológica como gobierno compartido. 5. Conclusiones. 6. Bibliografía.

Como citar: De La Peña Varona, A. y Mondragón Ruiz de Lezana, J. (2022). La Planificación hidrológica como gobierno compartido o el nexo entre sostenibilidad y federalismo. Polit. Soc. (Madr.) 59(1), 74627. https://dx.doi.org/10.5209/ poso.74627

\footnotetext{
Universidad del País Vasco (España)

E.mail: alberto.delapena@ehu.eus

2 Universidad del País Vasco (España)

E-mail: jaione.mondragon@ehu.eus
} 


\section{Introducción}

La Directiva Marco del Agua (DMA), aprobada en el año 2000, supuso un punto de inflexión en la política hidrológica de toda la Unión Europea, exigiendo un proceso de adaptación institucional a sus Estados miembros para ajustarse a unos contenidos y procedimientos ciertamente renovados. Así, desde el punto de vista procedimental, la aprobación de la norma comunitaria emplazó a los actores nacionales a acomodarse al modelo de la gobernanza multinivel, lo que aún hoy representa todo un desafío, por ejemplo, para el modelo territorial del Estado autonómico en cuanto implica esfuerzos continuos en aras a integrar la pluralidad territorial en las decisiones comunes, a través de los principios generales de cooperación y coordinación interadministrativa. Esto es, con la DMA, la política de aguas va a demandar nuevas prácticas de gobierno compartido, lo que plantea un reto constante para un sistema como el español, en el que frecuentemente se ha destacado un escaso desarrollo de dinámicas de colaboración intergubernamental, tanto por determinadas características institucionales como por una cierta cultura política y percepción territorial, en principio, poco acordes a los valores propios del federalismo.

Nuestra intención aquí es analizar los aspectos sustanciales de una dimensión concreta de la política de aguas, como es la planificación hidrológica (PH), planteándola como pauta de gobierno compartido en el modelo del Estado de las autonomías. Creemos que el interés de este análisis no radica tanto en describir la interacción entre las diferentes partes, cuanto en las implicaciones presentes y futuras de tales relaciones. Esto es, las claves que nos pueda ofrecer su estudio para comprender cómo funciona en la práctica el gobierno compartido en el caso español en una materia tan sensible como la planificación del agua, así como las posibles influencias de esos procesos en la manera de comprender el territorio por parte de los actores protagonistas de la gobernanza del agua. En este último aspecto, partimos de la presunción de que la proyección espacial de determinadas políticas públicas ligadas a la sostenibilidad ambiental habrá de acabar propagando entre sus protagonistas valores como la diversidad e interdependencia, haciéndoles, en definitiva, profundizar en el tránsito al federalismo.

En las líneas que siguen, por tanto, esbozaremos en primer lugar el modelo territorial sobre el que ese nuevo paradigma de PH se desarrolla, tratando así de comprender las oportunidades y limitaciones con las que los actores políticos se topan en su intento de implantar procedimientos y objetivos de la normativa comunitaria. A partir de ahí, profundizaremos en los aspectos de cambio que ha supuesto la DMA con respecto a la precedente política hidrológica para presentarla como una manifestación más del gobierno compartido (shared-rule), especialmente en los casos de demarcaciones intercomunitarias, donde las competencias se reparten entre la Administración General del Estado (AGE) y las comunidades autónomas (CC. AA.). Nos basaremos para ello en una previa revisión bibliográfica que toma como principales referencias tanto la literatura generada en torno a la cuestión territorial y el federalismo como sobre la política del agua en el caso español. Una revisión que se complementa con el análisis de documentación normativa para la cuestión de la PH.

\section{El Estado de las autonomías}

En los estudios de política comparada se traza muy frecuentemente una división entre centralización y descentralización atendiendo a cómo se distribuye el poder, desde una perspectiva territorial, en los diferentes sistemas políticos ${ }^{3}$. Partiendo de la base de que en esto como en tantas otras cosas los modelos puros no existen, el caso español parece haberse situado en cierta zona gris. Los especialistas lo han calificado en la mayoría de las ocasiones como modelo intermedio de descentralización que, sin ser nominalmente federal, sí parece mostrar un nivel acusado de descentralización con respecto a los sistemas unitarios e incluso cierta tendencia federalizante. En ese sentido, hablar del Estado de las autonomías supone afrontar cierta complejidad institucional y política como para justificar el interés de abordar un análisis específico en torno a cómo encajan modelos multinivel como el de la DMA en nuestro ordenamiento jurídico y, sobre todo, con qué posibilidades cuenta para implantarse exitosamente. A continuación, trataremos de caracterizar más detalladamente los aspectos institucionales y culturales de nuestro modelo territorial.

\subsection{El marco institucional: descentralización sin gobierno compartido}

De acuerdo al clásico planteamiento de Elazar (1987), el federalismo puede caracterizarse por la confluencia de dos dimensiones, autogobierno (self-rule) y gobierno compartido (shared-rule), que vendrían a ser las dos caras de una misma moneda y la gran alternativa al sistema unitario de distribución territorial del poder. Tal

La clasificación de los modelos de organización territorial ha estado presente desde los albores de la política comparada, donde se atendía fundamentalmente a la organización institucional de los diferentes Estados. En los últimos tiempos, el Regional Authority Index, diseñado y aplicado por Hooghe et al. (2016) a 62 países, ha sido, sin duda, una de las principales referencias, pues toma en consideración tanto la dimensión de autogobierno (self-rule) de las unidades territoriales constitutivas a nivel regional y local, como la de gobierno compartido (shared-rule) para evaluar el grado de descentralización de los diferentes casos. Más centrados en la primera de las dimensiones, podemos citar también los estudios de Dardanelli o Do Vale: el primero de ellos critica, desde el punto de vista conceptual, la inclusión del shared-rule como parte del fenómeno de la descentralización (2018; 275), y el segundo de ellos se centra específicamente en un análisis diacrónico del caso español (2021). 
sistema ofrece básicamente conjugar pluralidad y unidad, combinando tanto la posibilidad de gestionar competencias de manera autónoma por las entidades constituyentes regionales o intermedias, como la posibilidad de estas de concurrir a la toma de decisiones comunes (Elazar 1987: 84). Este principio federal resulta de especial interés cuando se trata de políticas ambientales que normalmente necesitan, aunque al mismo tiempo luchen contra ella, una autoridad compartida (Wälti, 2017).

Como se ha sugerido, es posible constatar cierta controversia acerca de la manera en que la literatura especializada aborda el sistema territorial español, un modelo descentralizado sobre la base de las CC. AA. y consagrado por la Constitución de 1978. Algunas lecturas han interpretado el proceso descentralizador que se plasma en el texto constitucional, fundamentalmente como un intento de retener a determinadas comunidades de especificidad etnoterritorial dentro del proyecto del Estado español — hold together — (Linz, 1997: 35-39). Ello marcará, en buena medida, una descompensación entre un acendrado autogobierno y una relativa ausencia de mecanismos institucionales para la toma de decisiones en común (Máiz, Beramendi y Grau, 2002: 388; Moreno, 2008: 144; Romero, 2020, 124 y ss.).

Abundando más en este análisis, se ha expuesto cómo ha sido frecuente la dinámica bilateral entre CC. AA. y órganos centrales del Estado, incentivada por un marco institucional que adolece de una serie de "déficits federales" sustanciados en los siguientes elementos: una interpretación extensiva de las competencias del Estado por la vía, normalmente, de la legislación de lo básico; la imposición del nivel europeo que determina ámbitos de gestión propios de las CC. AA. que, sin embargo, no tienen interlocución con las instituciones europeas, y una práctica ausencia de mecanismos para la integración de las CC. AA. en las decisiones comunes del Estado (Máiz, 2005: 163). En una línea similar, Moreno argumenta que hay cierta inconsistencia jurídica, resultado de la ambivalencia política del texto constitucional, la práctica de la bilateralidad en el traspaso de competencias y una grave confusión ideológica en la noción de Estado que ha dado lugar a una ausencia de vinculación de las CC. AA. con la toma de decisiones a nivel estatal y el mantenimiento de cierta supremacía centralista en las relaciones territoriales (2008: 157).

En suma, las dudas en torno al verdadero carácter federal del modelo territorial español provienen sobre todo de esta relativa ausencia de lo que podríamos llamar "gobierno compartido". La característica institucional del modelo español más comúnmente citada en este sentido es la ausencia de un Senado eficaz en su papel de cámara territorial. Efectivamente, el hecho de que las unidades representadas sean las circunscripciones electorales provinciales más que las CC. AA. en sí y la subordinación constitucional del Senado al Congreso de los diputados en el proceso legislativo deriva en la falta de un espacio político de codecisión (Romero, 2005: 65; Moreno, 2008: 158). Esto ha hecho que incluso cuando surgen dinámicas puramente territoriales, estas hayan sido resueltas en la cámara baja, donde los partidos nacionalistas periféricos han presionado para preservar sus intereses específicos (Colino, 2008: 582; Grau, 2010). Así, podemos encontrar interpretaciones que conciben el Estado de las autonomías como representativo más bien de un cierto modelo intermedio entre los extremos unitario y federal, precisamente por la relativa carencia de espacios formales de gobierno compartido (Marks et al., 2008; 2016).

Con todo y con ello, de este estado de cosas tampoco parece que pueda colegirse - al menos sin esa cierta controversia a la que aludimos - una total ausencia de la dimensión del gobierno compartido en el caso español. Determinadas interpretaciones destacan cómo, durante el proceso de consolidación del sistema territorial autonómico, la colaboración entre el Estado y las CC. AA. empezó a desarrollarse de manera espontánea para institucionalizarse progresivamente (García Morales et al., 2006), y cómo las relaciones intergubernamentales han cristalizado en instituciones diseñadas al efecto como las conferencias sectoriales. En ellas, en las últimas décadas se han consensuado acuerdos en torno a la distribución de recursos, se ha intercambiado información y se han llegado a pactos en torno a la cofinanciación de servicios públicos y la firma de convenios (Colino, 2008: 577). Esto es así hasta el punto de que parece generarse un sistema de cooperación y coordinación horizontal entre las CC. AA. desarrollado a través de mecanismos formales e informales que van proporcionando incentivos crecientes a los agentes político-administrativos para consolidarlo y reforzarlo (Colino, 2011). Consecuentemente, no sorprende que abunden los estudios que incluyen expresamente a España como un Estado de tipo federal o similar y reduciendo el hecho de su configuración como modelo "autonómico" a una mera cuestión nominal (Blanco Valdés, 2012; Bolleyer, 2006; Moreno, 2008) y sujeto, al igual que otros modelos descentralizados, tanto a tendencias centrífugas como centrípetas (Colino, 2020: 44-49).

\subsection{Elementos no materiales: cultura política federal y visión territorial}

Cuando se analiza el propio concepto de federalismo, es notoria la recurrencia de expresiones que aluden a elementos de "tensión", "disonancia" o "reconciliación" entre lógicas opuestas. Desde sus primeros teóricos, el federalismo se caracteriza por la conciliación de tendencias en permanente disputa, como son el localismo y la unión entre diferentes (Burguess, 2006: 9-25), que habría de resolverse sobre la idea del pacto - foedusque une lo particular y lo general en una entidad que se configura como soberana pero, al mismo tiempo, renunciando a una concepción transitiva del poder en aras a compartir, acordar, revisar y, en definitiva, limitar el propio ejercicio del mismo (Máiz, 2018: 483). 
Sobre esta base, la implantación de un modelo federal plantea un desafío que va más allá de un específico diseño institucional: en otros términos y dado que estamos hablando, en definitiva, de cómo se organiza el poder político, habremos de reparar en dónde halla este su legitimidad. En este caso, habría de ser en un universo compartido de valores, actitudes y elementos cognitivos, que se proyectara asimismo sobre la percepción del territorio en el imaginario colectivo, haciendo a la ciudadanía más consciente tanto de la diversidad como de la unidad de acuerdo a una concepción multiescalar del mismo. Recogeremos aquí este planteamiento añadiendo ese componente específico de la visión del territorio que entendemos forma parte del conjunto de elementos no materiales que componen el conjunto de precondiciones para el desempeño en clave federal. Reservaremos a este aspecto un espacio propio, en tanto interpretamos el carácter territorial como un elemento especialmente relevante de las políticas de sostenibilidad, entre ellas la política hidrológica. Como se verá, el contenido sustantivo, el marco institucional, los procedimientos y los resultados e impactos (outputs/outcomes) de esta se proyectan sobre el territorio y viceversa, desafiando las concepciones más extendidas de este y demandando nuevos marcos espaciales para su implementación y comprensión.

\subsubsection{Cultura política federal}

Entre las condiciones necesarias para un diseño institucional de carácter federal "exitoso", Simeon y Conwey destacan la coexistencia entre identidades subestatales o regionales con identidades y valores adscritos al nivel federal, una identidad dual que debería ser reflejada en las instituciones centrales que, a su vez, articularían sus procesos de toma de decisión mediante técnicas y procedimientos consociacionales ${ }^{4}$. Es una línea parecida la que sostiene Bednar al señalar la necesidad de una cultural federal que haga posible el mutuo ajuste entre niveles de gobierno y, en definitiva, una "federación robusta" a través de la adscripción ciudadana a unos determinados valores federales (2008, 187 y ss). Elazar (1987) apunta también a la necesidad de desarrollar un marco cognitivo, un thinking federal, que dote de sentido las acciones de los intervinientes en los procesos institucionales. Es a partir de ahí como, con ánimo sintético, Máiz demanda una revisión de las clásicas dimensiones empírico-normativas del federalismo para ser ampliadas y articuladas en modo coherente, de forma que todo sistema federal debiera de contar en su planteamiento con cinco condiciones diferentes: un gobierno multinivel garantizado mediante "salvaguardas institucionales", un régimen constitucional y de Estado de derecho en todos los niveles, un sistema descentralizado de partidos, experimentación y diferenciación de políticas públicas a lo largo de los diferentes niveles territoriales y una cultura política federal basada en el pacto, la negociación, la reciprocidad y el respeto mutuo (Máiz, 2018: 459).

Se conjugarían aquí, por tanto, dos aspectos básicos del análisis politológico: valores e intereses, de manera que la articulación concreta de los primeros orienta la formulación de los segundos. En otros términos, la formación y consolidación de una determinada distribución de valores o cultura política federal, basada en la empatía, la corresponsabilidad y las lealtades colectivas compartidas induce a una formulación de intereses en los procesos de negociación política referenciada en elementos de pertenencia común y reconocedora de la legitimidad de los objetivos de la contraparte. Sobre el modo en que esto podría eventualmente afectar a las decisiones públicas, sea en materia de política hidrológica u otra cualquiera, cabe recuperar el argumento de Fontaine (2015), que subraya la importancia de las ideas y del aprendizaje en el contexto institucional y en el proceso político, señalando que las conductas entre actores son producto de construcciones sociales, de percepciones y valores que conviene estudiar para explicar su comportamiento final.

Acerca de los efectos de la estructura territorial del Estado en la configuración valorativa de la ciudadanía, se han formulado hipótesis alternativas tanto en el sentido de que la descentralización genera un reforzamiento de la identidad colectiva adscrita a la comunidad plurinacional, como que, por el contrario, puede ahondar en las diferencias culturales de las minorías expandiendo los sentimientos de singularidad y, en última instancia, proporcionando incluso apoyos a movimientos secesionistas. La evidencia empírica provista por los estudios de opinión pública parecería aportar datos que corroboran una u otra hipótesis en función de la situación económica relativa de los casos analizados. En situaciones donde el nivel de desarrollo se encuentra por debajo de la media nacional, la descentralización institucional operaría en un reforzamiento del sentido de pertenencia al Estado; mientras que allí donde los indicadores económicos se sitúan por encima de la media, ese mismo proceso ahondaría en los "hechos diferenciales", minando el surgimiento de una identidad federal en ese sentido pluralista en el que se ha caracterizado anteriormente (Martínez-Herrera, 2010).

Retornando al caso español, la existencia de ese thinking federal no parece haber sido la nota predominante (Vallés 2016). Ya el análisis histórico muestra comúnmente la limitada extensión de las ideas federalistas, combatidas tanto por fuerzas liberales como por las conservadoras en la época del nation-building (Moreno, 2009: 123). Aún hoy, el propio sistema de partidos muestra una relativa debilidad de esas posiciones, toda vez que solamente en un sector de la izquierda de ámbito estatal pueden verse expresamente apoyadas en forma de programas y declaraciones públicas, mientras que el resto del arco parlamentario compone una horquilla que abarca desde posiciones de nacionalismo de Estado hasta proyectos nacionalistas periféricos. A estos dos

Citado en Burguess, 2006: 139 
bloques les une el hecho de que ambos propugnan un primordialismo identitario, bien de alcance estatal o bien a escala regional, en principio poco dado a una concepción territorial en clave de diversidad y variabilidad.

En esta línea, algunas de las explicaciones que apuntan a un relativo fracaso del gobierno compartido en el caso español inciden en aspectos relacionados con el modo en el que se interpretan mayoritariamente las relaciones territoriales. Desde una perspectiva general, la práctica del bilateralismo, en la que incurrirían tanto actores centralistas como "centrifugalistas autonómicos", compondría una explicación de por qué el modelo autonómico no ha evolucionado suficientemente en la multilateralidad y la cultura del pacto federal (Moreno, 2009: 121). Asimismo, y analizando el comportamiento de las instituciones de gobierno compartido del Estado autonómico, se han detectado problemas parecidos: una particular competición interpartidista, propia del modelo español, que subsume el factor territorial como otro aspecto más de la contienda político-electoral. También, cierta ausencia de cultura política federal, que llevaría a una dinámica en la que los nacionalismos español y periféricos se retroalimentan al redundar en una visión exclusivista de las competencias políticas (De la Peña et al., 2015; León y Ferrín, 2012: 75; Máiz, 2005: 164; Mondragón et al., 2016: 98; Romero, 2020, 137).

Tal visión parece coherente con determinados análisis de las relaciones intergubernamentales entre AGE y CC. AA. que han incidido en una cierta visión conflictiva de las mismas, sobre todo por parte del personal político frente a la clase funcionarial (Crespo, 2017: 486 y ss.). Y, asimismo, parece explicar los principales resultados obtenidos a través de los estudios de opinión pública. En ese sentido, se ha tendido a señalar tanto el escaso apoyo del federalismo frente a otras fórmulas territoriales (Lancaster, 1998: 258), como una cierta territorialidad de las percepciones, por la cual las regiones donde más claramente se politiza el hecho diferencial muestran una apuesta sostenida por la progresiva descentralización política del Estado (Pérez Castaños y García Rabadán, 2019).

\subsubsection{Visión territorial}

Habitualmente el territorio ha sido interpretado como una condición previa de aquellos fenómenos que han atraído la atención del análisis politológico y sociológico. Si bien desde la ciencia política se le ha concebido como uno de los elementos integrantes del Estado, junto con la población y la soberanía, en la mayor parte de las ocasiones no ha merecido mayor atención que la de ser el mero escenario físico en el que acontece el proceso político y el soporte material donde, si acaso, impactan las políticas públicas. En ese sentido el concepto de territorio se une inextricablemente a la existencia de una autoridad que circunscribe su soberanía sobre un espacio delimitado; es decir, representa la "extensión del poder del Estado" planteándose como "tecnología política", que abarca el conocimiento y el dominio sobre el propio espacio (Elden, 2013: 322 y ss.).

En las últimas décadas, la globalización y sus consecuencias han emplazado a repensar la soberanía y la propia cuestión territorial desde diversos ámbitos del conocimiento. En este sentido, el territorio se encuentra con la noción de escala, entendida como tamaño, magnitud o proporción. En él se desarrolla una determinada interacción entre elementos participantes y se despliegan procesos de variada naturaleza. Desde el estudio de la gestión integrada de los recursos naturales, comprender las dinámicas que afectan al agua, el aire o la biodiversidad nos aboca evidentemente a entender sus interacciones en diferentes dimensiones de rango variable desde lo micro a lo macro y, por tanto, a delimitar conceptualmente la escala y el propio análisis escalar. Por su parte, también la realidad institucional del sistema político determina un ámbito escalar autónomo y crucial en cuanto que define el área sobre el cual se aplican las jurisdicciones y se asignan determinados roles a agentes particulares. Partiendo de esa idea, la gestión óptima de los recursos naturales habría de implementarse a través de una ampliación de escalas fundamentada en una efectiva coordinación o visiones compartidas a través de los diferentes niveles institucionales (Lovell et al., 2002). En buena medida, este planteamiento es recogido ya en la Conferencia de Río de 1992, cuando advertía la necesidad de componer una agenda de acción que conciliase lo global y lo local. Dentro de este planteamiento, el factor institucional es indiscutiblemente el que se encuentra más cercano a nuestra perspectiva, al relacionarse con la delimitación de un ámbito político sobre el que se establecen bienes, recursos, derechos de apropiación, funciones y estructuras de autoridad (Ostrom, 2015).

Por otra parte, desde un enfoque de geografía crítica, se ha problematizado la cuestión de las escalas territoriales (local, regional, estatal, global...) concibiéndolas como constructo y producto de las relaciones económicas, políticas y sociales (Smith 2020, 113 y ss.). Definida en términos generales, la escala sería algo así como el nivel de resolución geográfica —el ámbito territorial, si se quiere - en el que un fenómeno es comprendido, interpretado o enmarcado, siendo incluso promovido por los distintos tipos de actores en según qué circunstancias: los agentes públicos o privados a la hora de invertir recursos; los movimientos políticos al movilizar sus apoyos; o, en definitiva, el global de los habitantes de un determinado espacio al desplegar su actividad e interacciones cotidianas (Agnew, 1997: 100-102). La procedencia marxista de la mayoría de los autores de esta corriente como Henri Lefebvre, David Harvey, Peter Taylor o Neil Smith les llevará a fijar su atención en la influencia ejercida en este proceso por las relaciones de producción. Se insiste en la idea de que el capitalismo hegemónico formula y reformula constantemente las escalas territoriales de acuerdo a sus propias necesidades e intereses, en un proceso de reajuste continuo para dar acomodo a las cambiantes demandas del mercado. Sería esta dinámica la que explicaría el actual proceso de "redimensionamiento" del Estado en un contexto de 
globalización del sistema productivo y financiero (Brenner, 2000 y 2004). Así, después de una época histórica de absoluta centralidad del Estado-nación a la hora de concebir el territorio, transitaríamos en la actualidad a una nueva época donde la coexistencia y el reordenamiento de escalas complejiza esa representación colectiva del territorio, en consonancia con una cosmovisión hegemónica de tipo neoliberal interesada principalmente en sortear el control público sobre el movimiento de los capitales.

Para nuestros intereses, el aspecto más relevante de este planteamiento es, sin duda, el hecho de que nos recuerda el carácter convencional, socialmente mediado, de la construcción del territorio en el sistema político, concibiéndolo como presupuesto, medio y resultado de las relaciones sociopolíticas. Pese a que pudiera parecer que son preexistentes y están ahí para ser utilizadas, las escalas son creadas como parte del planteamiento estratégico de los actores sociopolíticos (Herod, 1997: 163) y, aunque su aparición en cuanto objeto de reflexión teórica es relativamente reciente, sus expresiones prácticas no lo son y conectan con el problema que, en definitiva, estamos tratando aquí: el de la construcción del modelo territorial del Estado (Delaney y Leitner, 1997: 94; Herod, 1997). Los actores imaginan territorios, proyectan su poder sobre ellos y promueven particulares sistemas de jerarquización entre las distintas escalas.

En el caso español, como en el resto de los países, la construcción del territorio es deudora del propio proceso histórico. Lo que sí es más idiosincrático del caso español es que las estrategias de nation-building coinciden con un periodo de crisis sistémica, pérdida de influencia internacional y movimientos por la regeneración; en un tiempo, además, en el que la no coincidencia geográfica de los polos de desarrollo económico y político llevan al surgimiento de nacionalismos periféricos (Álvarez Junco, 2001: 593 y ss.). Los orígenes de la política hidrológica se sitúan en esas mismas coordenadas históricas representando la visión de una parte del proyecto regeneracionista que apostaba por el aprovechamiento máximo de los recursos hidrográficos y la construcción de grandes pantanos que extendiesen el regadío, facilitasen la generación hidroeléctrica y contribuyesen a paliar el "secular" socioeconómico español. Es el planteamiento de Lorenzo Pardo, sin duda la primera figura de relevancia en la historia de la política hidrológica y, en cierto modo, padre de una administración hidrológica que acuñará una visión del territorio diferente, basada en cuencas hidrográficas o, lo que es lo mismo, escalas determinadas por una realidad natural como es el río. Llegado el momento, el trazado del mapa autonómico reflejará el surgimiento de esos nacionalismos periféricos y se llevará por delante determinadas formas de entender el territorio y la identidad territorial, casi siempre originarias de esa parte de España que no tenía mayor poder para dar una expresión política a sus planteamientos (Moreno, 2008: 80-87), pero habría de seguir conviviendo con el mapa hidrológico atento a esa otra forma de concebir el espacio.

En este contexto general, categorías como lo local, regional, nacional, etc. son entendidas de distinta manera por según qué actores políticos que tienden a desarrollar sus respectivas lecturas del territorio en función de visiones ideológicas, planes estratégicos o realidades organizativas. Partiendo de esa base, los actores políticos maximizan sus intereses y apoyos a la vez que recurren a tácticas de inclusión y exclusión, provocando disonancias en el lenguaje y formas encontradas de enjuiciar una misma realidad o política pública. Esto es especialmente visible en contextos donde concurren distintos sentimientos de identificación nacional subjetiva: particularmente, la forma de entender lo regional o nacional varía en función de la adscripción políticoideológica, lo mismo que cambian las nociones de centro y periferia. Se observa con nitidez en la utilización diferenciada que hacen los actores políticos de tales términos en el debate público 5 .

Desde esta óptica, las políticas públicas vendrían condicionadas por una determinada configuración territorial sobre la que, a la postre, proyectarían su resultado contribuyendo a una eventual rearticulación. Considerando la política pública desde la perspectiva de las policy networks, podemos presuponer una determinada confluencia de actores diversos en un espacio común en el que se desarrollan sus interacciones. La configuración del territorio determina por tanto la delimitación de las dimensiones horizontal y vertical y, por añadidura, el enmarque del problema colectivo, el número y la heterogeneidad de los actores intervinientes, así como el estilo de la política pública.

Todas estas consideraciones adquieren un especial relieve en una política sectorial como la hidrológica, donde se evidencia la existencia de ámbitos de decisión propios que no aparecen en otras áreas de intervención pública y que, por ello, necesitarían ser relegitimados en un contexto como el actual de creciente cuestionamiento de las diferentes territorialidades. El hidrológico es, en ese sentido, "un terreno especialmente significativo para el debate del ajuste espacial" (Del Moral y Hernández-Mora, 2016: 3), toda vez que, a pesar de la aparente objetividad de los elementos que componen el ámbito sobre el que se tomas las decisiones — la

Ello se plasma en la forma de describir o recrear gráficamente el país en los informativos de los medios de comunicación públicos, como suele suceder con el ejemplo recurrente del mapa del tiempo (https://www.lavanguardia.com/local/pais-vasco/20130326/54370693095/etb-euskal-herriamapa-tiempo.html, consultado el 11 de diciembre de 2021) o al plantear determinadas actuaciones públicas como la construcción del Tren de Alta Velocidad, que representa para el nacionalismo vasco una especie de metro de la CAPV (entendida como metrópoli vasca o Euskal Hiria), al tiempo que para los partidos de ámbito estatal se entiende fundamentalmente como la forma de conectar Madrid con la frontera de Irún, Bilbao y su puerto. Son dos lecturas muy diferentes de una misma intervención pública, inspiradas por formas alternativas de representar y recrear el territorio en el debate público y el imaginario colectivo y, asimismo, dos interpretaciones que nos recuerdan que un mapa es, en acertada expresión de Sergio del Molino, “...un instrumento que sirve para que su autor se encuentre y se defina en relación con el espacio. Una forma de conocimiento y de dominio a la vez" $(2018,279)$. 
cuenca hidrográfica—, también está sujeto al impacto de procesos sociopolíticos que influyen sobre su definitiva delimitación.

\section{La Directiva Marco del Agua: gobernanza multinivel en el tránsito a la sostenibilidad}

La aprobación de la DMA en el año 2000, y su correspondiente trasposición a través del texto refundido de la Ley de Aguas ${ }^{6}$, supuso un cambio trascendental en el contenido de la política hidrológica al replantear los objetivos generales de las políticas del agua y marcar la sostenibilidad como objetivo prioritario (Arrojo 2011; Cirone, 2013; Espluga et al., 2011; Estevan, 2006; Font y Subirats, 2010; La Roca, 2011). Asimismo, y más allá de esta dimensión sustantiva, la aprobación de la DMA tuvo también el efecto de situar la política del agua dentro del paradigma de la gobernanza multinivel, según el cual, las instituciones comunitarias pasaron a ejercer una función normativa necesariamente recogida por el ordenamiento jurídico de los Estados miembros e implementada por las instituciones de sus diferentes niveles territoriales. En suma, se avanza hacia la dispersión de la autoridad política tanto dentro del Estado como más allá de este (Hooghe et al., 2021: 197).

Todo ello impuso un cambio al modo de hacer políticas del agua, concretándose, entre otros, en el mandato comunitario que establece la necesidad de modelos participativos en la toma de decisiones y de coordinación interinstitucional. En el caso español, esta necesidad de coordinación ya estaba presente en la Ley de 1985 (VV.AA. 1999: 510), pero es la DMA la que va a establecer tal aspecto como un mandato de la nueva política del agua (especialmente, en los artículos 3 y 13) de la que se deriva, por ejemplo, la necesidad de constituir comités de autoridades competentes en los organismos encargados de la $\mathrm{PH}$. El relativo fracaso de estos evidencia que aún queda pendiente la eficaz integración de las diferentes administraciones implicadas en las demarcaciones (Del Moral y Hernández-Mora, 2016: 14).

El contexto político en el que se va a incorporar la nueva norma europea al ordenamiento jurídico español va a estar marcado en su fase inicial por el nuevo proyecto de Plan Hidrológico Nacional (PHN), impulsado por el segundo gobierno de José María Aznar y fuertemente contestado por una parte importante de las CC. AA. y actores de la sociedad civil, principalmente a cuenta de los trasvases entre cuencas y la fuerte inversión en infraestructura hidráulica que acarrea. Es entonces cuando se asiste al surgimiento de una denominada "nueva cultura del agua" que opone objetivos ambientalistas a la clásica concepción desarrollista de la política hidráulica (Aceros y Domènech, 2010: 14-15) y que encuentran precisamente en la DMA un referente normativo al que aferrarse en su oposición al PHN7 .

En todo caso, a partir de la aprobación de la DMA, y su correspondiente trasposición, se implanta de forma indiscutible un nuevo paradigma de $\mathrm{PH}$, basado en el concepto de demarcación hidrográfica (que incluye aguas subterráneas, costeras y de transición junto con las fluviales en el dominio público hidráulico). A partir de este nuevo paradigma, la planificación se desgrana en diferentes ciclos orientados a la consecución ya en 2015 de un buen estado ecológico de las masas de agua de la UE (Cirone, 2013: 76). Este modelo fue adaptado tardíamente al caso español, y con escasa voluntad política a juicio de algunos autores (Hernández Mora, 2012), lo cual no impide que, para enero de 2016, se hubieran aprobado 16 planes de demarcación, en su segundo ciclo, incluyendo las cuencas intercomunitarias más importantes de la península. Actualmente, se encuentra en fase de elaboración la planificación correspondiente al tercer ciclo, que deberá determinar la gestión de las masas de agua durante el periodo 2021-2027.

Consecuentemente, se puede sostener que la DMA muestra ya una larga trayectoria a partir de la cual cabe comenzar ya a evaluar su potencial transformador en una doble dirección. Primero, la relacionada con el contenido sustantivo de la política, esto es, si verdaderamente ha cuajado la apuesta por la sostenibilidad como principal objetivo de la planificación y gestión en materia de aguas y, segundo, el establecimiento de una nueva forma de proceder basada sobre todo en el paradigma de la gobernanza multinivel y, concretamente, en el principio de cooperación y coordinación entre las diferentes administraciones territoriales ${ }^{8}$. Si es así, ¿cómo se ha proyectado sobre el caso español? Esa será la cuestión a la que dirigiremos nuestra atención en los epígrafes subsiguientes, fijándonos en las connotaciones particulares del Estado de las autonomías tanto desde la perspectiva de su configuración institucional como de la cultura política de su ciudadanía y élites políticas.

RDL $1 / 2001$, de 20 de julio.

Buena parte de estos conflictos vendrán determinados además por intereses territoriales diversos como corresponde a un país con importantes desequilibrios hídricos internos, de los cuales las CC. AA. serán abanderadas, convirtiéndose frecuentemente en policy entrepreneurs (Font y Subirats, 2010: 10). Lo cierto es que en 2005, el PHN fue modificado parcialmente en sus aspectos más polémicos después de la llegada al gobierno del PSOE, que plantea el denominado Programa AGUA y que recoge algunos de las demandas de los actores más conservacionistas (Espluga et al., 2010).

8 Más allá de las preguntas que, desde una perspectiva académica, podamos plantearnos en este ámbito, lo cierto es que la DMA ya ha pasado recientemente por su propio proceso de evaluación (fitness-check), en el que la Comisión Europea ha tratado de calibrar su implantación basándose en determinados criterios: relevancia de sus objetivos, eficacia y eficiencia en su consecución, coherencia interna y externa con otras políticas y valor añadido europeo (Martínez-Hernández, 2019). La valoración final ha desencadenado numerosas críticas entre las organizaciones conservacionistas, dirigidas hacia la administración hidrológica y motivadas fundamentalmente por la escasez de resultados positivos respecto a los contenidos y objetivos de la DMA (https://www.iagua.es/noticias/wwf/comision-europea-vuelve-suspender-espana-aplicacion-directiva-marco-agua-0) 


\section{La planificación hidrológica como gobierno compartido}

\subsection{La cuenca hidrográfica como ámbito territorial de la política de aguas}

Como se ha dicho con anterioridad, en España la política hidrológica se ha consolidado desde hace muchos años sobre la existencia de un ámbito territorial propio, no coincidente con la delimitación político-administrativa ${ }^{9}$. Desde los años veinte del siglo pasado existen las confederaciones hidrográficas que se constituyen como gestores de la política de aguas sobre el referente geográfico de la cuenca, concepto recurrente en hidrología que comprende la superficie territorial vertiente a un curso de agua principal hasta su desembocadura en el mar incluyendo los distintos afluentes, ríos, arroyos e, incluso, aguas subterráneas ${ }^{10}$. En este sentido, la política hidrológica desde el primer momento se fundamenta en unidades geográficas consideradas en una dimensión escalar propia y referenciada al entorno biofísico.

No vamos a desarrollar aquí una visión histórica de la evolución de la política de aguas ${ }^{11}$, pero sí diremos que esta configuración inicial sobre la realidad de la cuenca ha venido manteniéndose independientemente de los diferentes modelos territoriales, incluyendo el autonómico instaurado en 1978. De hecho, una de las principales funciones de la ley de aguas que se aprobaría en 1985 fue precisamente la de aclarar y delimitar las competencias de las diferentes administraciones territoriales en materia hidrológica, toda vez que esta tomaba una referencia territorial distinta a la propia de las CC. AA. ${ }^{12}$. Así, un aspecto absolutamente nuclear de esta política sectorial viene dado por el hecho de que la legislación establece una diferenciación básica entre cuencas intercomunitarias e intracomunitarias, según la totalidad del curso de agua principal transcurra íntegramente o no por el territorio autonómico. Esto va a suponer que, dependiendo de las particulares situaciones geográficas, buena parte de los actores autonómicos perciban agravios comparativos que traten de compensar con reformas estatutarias que cuestionan el principio de unidad de cuenca y que han sido motivo de airada discordia (Fanlo, 2010; Sánchez Martínez, 2011).

En cualquier caso, cada cuenca hidrográfica, ya sea intra o intercomunitaria, se dota de un ente administrativo que se encarga de desarrollar tanto la planificación de las diferentes demarcaciones como su gestión a nivel de adjudicación de concesiones, prevención del riesgo de inundaciones, policía de aguas, etc. En el caso de las cuencas intracomunitarias, son las administraciones autonómicas las que han determinado el órgano de gestión que, en un buen número de ocasiones, ha tomado la forma de agencias autonómicas del agua, como es el caso de Galicia, Euskadi o Catalunya ${ }^{13}$. Para las cuencas intercomunitarias, el organismo designado han sido las confederaciones hidrográficas, de larga tradición en nuestra política hidrológica y que, según algunas voces, difícilmente pueden, por su naturaleza organizativa, evolucionar hacia una gestión integral de los recursos hídricos que encaje en las políticas medioambientales (García López, 2016: 100). Con mayor o menor éxito, y de acuerdo a los valores planteados por la propia Directiva Marco de Aguas de la UE, la labor de estos organismos de cuenca se aborda sobre criterios participativos, integrando a todos los actores territoriales, así como a la ciudadanía en general a través de participación de base asociativa o individual, en los respectivos consejos del agua de la demarcación.

Entendido esto, no resulta extraño que quizá donde las tensiones territoriales se plantean de una manera más obvia es en el caso de los organismos de cuencas intercomunitarias, en cuyos consejos del agua se plasman a menudo conflictos de intereses entre las CC. AA. y el Estado, si bien reservando siempre la capacidad decisoria para la Administración central, a la que en definitiva las confederaciones pertenecen en calidad de organismos autónomos adscritos al ministerio con competencias ambientales. Por ello, la historia de la política hidrológica en las décadas recientes vendrá marcada en buena medida por los intentos de las élites autonómicas de ganar competencias y protagonismo, así como por su resistencia frente a las decisiones del Estado en materia de redistribución de recursos hídricos. Este aspecto ha aparecido con especial virulencia en los proyectos de PHN, pero también en la enunciación de las competencias estatutarias en los sucesivos procesos de reforma y en la planificación de cada una de las cuencas intercomunitarias (Del Moral y Hernández-Mora, 2016: 14-16).

Independientemente de las soluciones institucionales concretas a los desafíos planteados por la gestión del agua, lo que vemos aquí es una utilización alternativa de la cuestión escalar para reconfigurar el territorio sobre una base diferente: una unidad geográfica delimitada en función de un recurso natural vertebrador de la misma,

Trabajos como los de Indset (2018) y OCDE (2011) han puesto de manifiesto cómo, a la hora de implementar la normativa comunitaria en el caso de la política de agua, la existencia de un modelo unitario no necesariamente tiene que significar la concentración de competencias en el nivel nacional, siendo más bien el caso que el reparto de competencias se establece teniendo en cuenta factores geográficos, medioambientales y económicos.

10 La DMA hace suya la cuenca hidrográfica como referente territorial para la gestión del agua, definiéndola en su artículo 2.13 como "la superficie de terreno cuya escorrentía superficial fluye en su totalidad a través de una serie de corrientes, ríos y, eventualmente, lagos hacia el mar por una única desembocadura, estuario o delta". La propia DMA reconvierte las cuencas en "demarcaciones hidrográficas" que, aparte de los ríos propiamente dichos, abarcan las aguas costeras y los acuíferos presentes en el territorio por donde discurre el curso fluvial principal.

Véase al efecto Pérez Díaz et al., 1996; Del Moral y Hernández Mora, 2016.

12 Por lo demás, la ausencia de correlación entre el modelo político-territorial de un país (unitario o federal) y el mapa institucional para la política del agua es un fenómeno relativamente frecuente (OCDE, 211; García López, 2016; Indset, 2018).

13 Augas de Galicia, URA y Agencia Catalana del Agua respectivamente. 
como es el ecosistema fluvial. Es este el que emplaza al legislador a delimitar ámbitos de gestión específicos y diferenciados del modelo político-administrativo con el cual no pocas veces entra en colisión. Aspecto este, por otra parte, recurrente en los problemas medioambientales que añaden otra dimensión al "mix" multinivel, en tanto se desarrollan en sistemas naturales territorialmente complejos donde se superponen fronteras naturales e institucionales (Wälti, 2017: 165) ${ }^{14}$.

\subsection{La planificación hidrológica en el caso español: entramado de actores e interacciones (instituciones y dinámicas)}

Como se dijo anteriormente, el modelo diseñado por la DMA fue adaptado tardíamente y con escasa voluntad política al caso español, si bien se han aprobado a lo largo de la última década dos ciclos sucesivos, correspondientes a 16 planes de demarcación que incluyen la totalidad de las demarcaciones intercomunitarias de la península. En ello se ha seguido el procedimiento pautado por la normativa correspondiente: en este caso, el RD 907/2007 de 7 de julio, por el que se aprueba el reglamento de la PH, cuyo Título II, Sección $3^{\text {a }}$ enumera las etapas preceptivas a través de las cuales se despliega cada uno de los ciclos planificadores que son básicamente los siguientes: la preparación de los documentos iniciales de la planificación, la elaboración del denominado Esquema de Temas Importantes (ETI) y la redacción del Plan Hidrológico propiamente dicho. Siendo el ETI algo así como el compendio de temas a regular por el plan, se redacta en principio una versión provisional del mismo que se somete a consulta pública recibiendo alegaciones de actores implicados de diversa naturaleza público-privada. La versión definitiva del ETI alimenta el documento definitivo de planificación que es finalmente aprobado por los consejos del agua de la demarcación y del Estado.

Obviamente, la composición de los consejos participativos de las diferentes cuencas, así como el análisis de los procesos de consulta e información pública de las distintas etapas del proceso planificador ${ }^{15}$ informan sobre el entramado y el carácter de las interacciones de la policy network estructurada en torno a cada una de las demarcaciones y participada por un número de actores público-privados, entre los que se cuentan administraciones de variable alcance territorial, asociaciones conservacionistas, grupos de interés como federaciones de regantes, corporaciones económicas, sindicatos, etc. En las cuencas intercomunitarias, una parte sustancial de ese sistema de gobernanza es lógicamente la compuesta por las relaciones entre administraciones territoriales de rango autonómico y la AGE, ámbito en el cual se despliega la noción de gobierno compartido propia de todo sistema descentralizado y, como vimos, explícitamente alentada por la propia normativa comunitaria de aguas ${ }^{16}$.

A pesar de este sistema de gobierno multinivel, la política hidrológica muestra una fuerte influencia de la Administración central, especialmente visible en el ámbito de la $\mathrm{PH}$ de las cuencas intercomunitarias y probablemente derivada de la división competencial operativa en este ámbito sectorial. Además del papel protagonista de las confederaciones hidrográficas como organismos de cuenca, es al Gobierno central a quien le corresponde la aprobación final de los planes de cuenca mediante real decreto, incluso en los casos de cuencas intracomunitarias, si bien la tendencia en este ámbito parece haber mostrado más la celebración de acuerdos con las administraciones hidráulicas regionales que un protagonismo real en la elaboración (Gallego et al., 2020: 346). Más aún, la competencia de elaboración de planes hidrológicos nacionales, que puede llevar aparejada la posible propuesta de trasvases intercuencas, reserva para el Gobierno central un papel decisorio final acorde con el sistema de distribución competencial diseñado en la Constitución de 1978 (art. 149). Sin embargo, es probablemente demasiado preponderante en un ámbito donde los intereses territoriales confluyen a menudo con la elaboración de discursos de sesgo identitario que enmarcan los conflictos en presuntos y seculares agravios regionales y/o nacionales.

A este factor relacionado con el reparto de poderes, podríamos añadir otro más relacionado con cierta manera de entender la pluralidad territorial, la composición del Estado y acaso el propio valor del recurso hídrico. Nos referimos a cierta ambivalencia que parece haberse dado en la delimitación por cuencas en el caso español y que determinaría que estas no hayan sido interpretadas tanto como ámbitos de concurrencia o de gobierno compartido, sino más bien como delimitaciones excluyentes que abortan la posibilidad de aplicar un paradigma de gestión integrada de los recursos hídricos al no reconocerse la interdependencia multinivel ni el enfoque coordinado de todas las partes implicadas (García López, 2016: 209). Esta idea es especialmente interesante

\footnotetext{
14 También es cierto que la aparente neutralidad político-social de la delimitación de las cuencas, en cuanto realidad definida por parámetros naturales, no lo es tanto si nos acercamos a ella en detalle. En ese sentido, lo que dijimos anteriormente sobre la mediación social de las escalas se reproduce, dada la influencia de factores históricos, económicos, sociales y políticos en la delimitación de su alcance territorial y como, por otra parte, ha sido puesto de relieve por el concepto de "territorio hidrosocial” aplicado al ámbito específico de la política hídrica (Del Moral y Hernández-Mora, 2016: 3). Desde una perspectiva más amplia, esta idea conecta asimismo con ciertos planteamientos que nos avisan acerca de la "reescritura" de la naturaleza (Swyngedouw, 2015) o de la hibridación entre elementos naturales y culturales propia del Antropoceno (Arias Maldonado, 2016).

Por ejemplo, las alegaciones a los ETI y su posterior respuesta por parte de los organismos de cuenca.

16 Del contenido de esta se deriva la propia composición de un Comité de Autoridades Competentes que informaría del proceso planificador, facilitando la cooperación entre las administraciones implicadas, y que parece no haber tenido aún un recorrido suficiente como para generar un impacto real en el contenido de la política hidrológica.
} 
en cuanto alude a factores no directamente derivados del marco institucional, sino a la forma en que este se asimila e interpreta.

\section{Conclusiones}

Como hemos ido viendo a lo largo de estas líneas, la PH en las demarcaciones intercomunitarias se ha desarrollado en un contexto multinivel como consecuencia de la aplicación de la DMA, que ha forzado tanto un cambio sustantivo como procedimental en las políticas del agua españolas. De esta manera, las confederaciones hidrográficas han asumido la gestión de un ámbito territorial, como es la propia cuenca, que por su propia naturaleza pone en común a diferentes actores territoriales, remedando los principios del gobierno compartido propios del federalismo. Tal proceso, no obstante, parece haber sido asumido de una manera incompleta de acuerdo a la mayor parte de la literatura especializada, lo cual entronca con la relativamente escasa presencia de elementos de cooperación intergubernamental y horizontalismo en el caso español. Quizá el ya referido fracaso del Comité de Autoridades Competentes pueda ser una ilustración de todo esto.

En cualquier caso, el de la implantación de la DMA es un proceso en marcha cuyas consecuencias pueden trascender su ámbito sectorial: si parte de las políticas públicas han de gestionar bienes comunes y asegurar una determinada transición al paradigma de la sostenibilidad, la noción de escala o de territorio deviene central tanto para el diagnóstico como para la acción. La PH compone un excelente ejemplo de cómo la naturaleza del bien público a proteger —en este caso el buen estado de las masas de agua y los ecosistemas fluviales - desafía el modelo territorial imperante, pero también se dan casos similares en otras actuaciones orientadas hacia la sostenibilidad, como la lucha por la preservación de la biodiversidad, la protección del paisaje o la ordenación del territorio, donde se construyen ámbitos espaciales alternativos como biorregiones, cuencas visuales, áreas funcionales, etc.

Desde nuestra perspectiva, por tanto, el valor de la política de aguas pasa fundamentalmente por el hecho de que sitúa la cuestión del territorio y la superposición de escalas en primer plano, lo que, unido a los objetivos sustantivos y procedimentales del ordenamiento jurídico superior — esto es, la DMA — emplaza a los actores a interactuar y pensar en clave federal, favoreciendo una visión alternativa del territorio. De esta forma, la necesidad de gestionar recursos naturales fuerza a considerar los necesarios flujos sobre una base espacial diferente, subrayando relaciones de dependencia que forjan intereses comunes y, a la postre, conducen a una visión alternativa de ese o esos vínculos de adscripción primordiales relacionados con las formas de organización territorial, ya sean unitarias o descentralizadas. Emergen así nuevos territorios que adquieren expresión político-administrativa sobre la base biofísica del espacio natural, el río o la cuenca hidrológica.

Consecuentemente, así como se evidencian las dificultades para la implantación de la DMA, se puede vislumbrar, por otra parte, el potencial de esta y otras políticas de sostenibilidad para contribuir a establecer unas relaciones diferentes entre los actores implicados y asentar una visión del territorio más compleja, conectada con la noción de "geometría variable" (Romero, 2020: 147), por la cual las fronteras político-administrativas cambian en función del bien común a preservar o gestionar. Se trataría de desescalamiento y reescalamiento continuos para abordar las demandas de la sostenibilidad ambiental, de manera que la adquisición de una nueva concepción del marco Estado-nacional, a partir de identidades superpuestas, facilitaría y a la vez sería consecuencia de la propia gestión de la sostenibilidad.

Aquí es donde encaja la noción de gobierno compartido y la cultura política federal, entendida como aquel marco interpretativo que integra lo común y lo particular, que acepta la diversidad sin desentenderse de lo común y que prioriza la toma de decisiones consensuada. En alguna ocasión se ha planteado el federalismo como biomímesis, incidiendo en lo que tiene de relaciones simbióticas, cooperativas, diversificadoras, etc. (Máiz, 2013: 98; 2018, 471 y ss.): es hora de afianzar esta propuesta teniendo en cuenta que el tránsito a la sostenibilidad no admite demora. Podríamos sostener por tanto que, como se sugiere en el título de este trabajo, federalismo y sostenibilidad comparten un nexo común. En el caso español ya existe, con sus limitaciones, un marco institucional propio del federalismo: el reto de avanzar hacia ese thinking federal podría pasar por una cierta práctica de la integración de la diversidad y la construcción de consensos, a través de la gestión de los bienes comunes.

\section{Bibliografía}

Aceros, J. C. y M. Doménech (2010): “La mancomunidad de política hidrológica española. Sectores y trayectorias políticas en Internet”, REIS: Revista española de investigaciones sociológicas, 132, pp. 11-34.

Agnew, J. (1997): "The dramaturgy of horizons: geographical scale in the 'Reconstruction of Italy' by the new Italian political parties, 1992-1995", Political Geography, 16(2), pp. 99-121.

Álvarez Junco, J., (2001): Mater dolorosa: la idea de España en el siglo XIX. 1 edn. Barcelona, Taurus.

Arias Maldonado, M. (2016): "El giro antropocénico. Sociedad y medio ambiente en la era global”, Política y Sociedad, 53(3), pp. 795-814. 
Arrojo, P. (2011): "Tiempos de cambio en materia de gestión de aguas", Quaderns de la Mediterrània = Cuadernos del Mediterráneo, 16, pp. 219-228.

Blanco Valdés, R. L (2012): Los rostros del federalismo, 1 edn. Madrid, Alianza Editorial.

Bolleyer, N. (2006): "Intergovernmental Arrangements in Spanish and Swiss Federalism: the Impact of Power-Concentating and Power-Sharing Executives on Intergovernmental Institutionalization”, Regional \& Federal Studies, 16(4), pp. 385-408.

Bednar, J. (2008): The Robust Federation. Principles of Design, Cambridge University Press.

Brenner, N. (2004): "Urban Governance and the Production of New State Spaces in Western Europe, 1960-2000“, Review of International Political Economy, 11. pp. 447-488.

Brenner, N. (2000): “The Urban Question as a Scale Question: Reflections on Henri Lefebvre, Urban Theory and the Politics of Scale", International Journal of Urban and Regional Research, 24, pp. 361-378.

Burgess, M. (2006): Comparative Federalism: Theory and Practice, Abingdon, Taylor \& Francis.

Cirone, M. (2013): "Una introducción a los aspectos más destacables del marco jurídico e institucional sobre la gestión del agua en España”, Derecho y ciencias sociales, 9, pp. 69-89.

Colino, C. (2020) "Instituciones y dinámicas territoriales en el Estado Autonómico: una panorámica de los debates y enfoques existentes”, en F. J. Fuentes et al. (eds.), Las transformaciones territoriales sociales del Estado en la edad digital, Madrid, Centro de Estudios Políticos y Constitucionales, pp. 29-54.

Colino, C., S. León y M. Ferrín (2012): La práctica de la cooperación intergubernamental en España, 1 edn. Madrid, Centro de Estudios Políticos y Constitucionales.

Colino, C. (2011): "Federalismo horizontal en el Estado Autonómico. La evolución de los mecanismos de cooperación horizontal en España", Revista Cuadernos Manuel Giménez Abad, 2, pp. 1-13.

Colino C. (2008): "Spanish model of devolution and regional governance: evolution, motivations and effects onpolicy making", Policy and Politics, 36(4), pp. 573-86.

Crespo, J. (2017): “Coordinación intergubernamental en España vista por la alta función pública del Estado”, Política y sociedad, 54(2), pp. 469-496.

Dardanelli, P., (2018) : “Conceptualizing, Measuring, and Mapping State Structures-with an Application to Western Europe, 1950-2015", Publius The Journal of Federalism, 49. pp. 271-298.

De La Peña, A., A. Elizondo, P. Juaristi, J. L. Mokoroa y J. Mondragón (2015): "Las conferencias sectoriales (2001-2012): Dinámica de funcionamiento y valores y percepciones de los agentes políticos y técnicos”, Gestión y análisis de politicas públicas, 14, pp. 23-41.

Del Molino, S., (2018): Lugares fuera de sitio, Barcelona, Espasa.

Del Moral, L. y N. Hernández-Mora (2016): "Nuevos debates sobre escalas en política de aguas. Estado, cuencas hidrográficas y comunidades autónomas", Ciudad y Territorio. Estudios Territoriales, 190, pp. 563-583.

Delaney, D. y H. Leitner (1997): “The Political Construction of Scale”, Political Geography, 16. Pp. 93-97.

Do Vale, H.F. (2021): "Four decades of territorial distribution of power in Spain: a measurement of subnational autonomy (19742018)", REIS: Revista Española de Investigaciones Sociológicas, 173, pp. 3-26.

Elazar D. J. (1987): Exploring federalism, Toscaloosa, University of Alabama Press.

Elden, S. (2013): The birth of Territory, London, University of Chicago Press.

Espluga, J., A. Ballester, N. Hernández-Mora y J. Subirats (2011): "Participación pública e inercia institucional en la gestión del agua en España", Reis: Revista española de investigaciones sociológicas, 134, pp. 3-26.

Estevan, A. (2006): "La Directiva Marco del Agua Europea: un profundo cambio de perspectiva en la gestión del medio acuático", Temas para el debate, 137, pp. 47-48.

Fanlo, A. (2010): "Las competencias del Estado y el principio de unidad de gestión de cuenca a través de las Confederaciones Hidrográficas", Revista de administración pública, 183, pp. 309-334.

Font, N. y J. Subirats (2010): "Water management in Spain: The role of policy entrepreneurs in shaping change", Ecology and Society, 15(2), pp. 21.

Fontaine, G (2015): El análisis de politicas públicas: conceptos, teorías y métodos, 1 edn, Barcelona, Anthropos.

Gallego Corcoles, I., N. Garrido, E. González y F. Delgado (2020): "Caudales ecológicos y otros conflictos ambientales en la reciente jurisprudencia derivada de la planificación hidrológica en España”, Revista Vasca de Administración Pública.HerriArduralaritzako Euskal Aldizkaria, 117, pp. 341-386.

García López, M. (2016): Retos para la gobernanza del agua: el caso de las cuencas mediterráneas andaluzas, Tesis doctoral inédita, Universidad de Málaga.

García Morales M. J., J. A. Montilla y X. Arbós (2006): Las relaciones intergubernamentales en el estado autonómico, Madrid, Centro de Estudios Políticos y Constitucionales.

Grau Creus, M. (2010) : “The Spanish Lower Chamber of Parliament: An Intergovernmental Arena? The Representation and Integration of Territorial Interests within the Congreso de los Diputados", en Legislatures in Federal Systems and Multi-level Governance, Baden-Baden, Nomos.

Hernández-Mora, N. (2012): “La planificación hidrológica y la Directiva Marco del Agua ¿Hemos avanzado?”, Ríos con vida AEMS, 88, pp. 40-44.

Herod, A. (1997): "Labor's spatial praxis and the geography of contract bargaining in the US east coast longshore industry, 195389", Political Geography, 16, pp. 145-169.

Hooghe, L., G. Marks y A. Schakel (2021): Multilevel governance, Oxford University Press, Chapter 11, pp. 193-210.

Hooghe, L., G. Marks, A. H. Schakel, S. Niedzwicecki, S. Chapman Osterkatz y S. Shair-Rosenfield (2016): Measuring Regional Authority. A Postfunctionalist Theory of Governance, Volumen I, Oxford, Oxford University Press.

Indset, M. (2018): “ Building bridges over troubled waters: Administrative change at the regional level in European, multilevel water management”, Regional \& Federal Studies, 28, pp. 1-22.

La Roca, F. (2011): "Del productivismo a la recuperación de los ecosistemas. La difícil transición de la política del agua en España”, Revista Iberoamericana de Economía Ecológica (REVIBEC), 16, pp. 99-112. 
Lancaster, T. D. (1998): "Nacionalismos, regionalismo e instituciones estatales: una evolución del caso español a partir de las opiniones de los ciudadanos” en R. Agranoff y R. Bañón (eds.) El Estado de las Autonomías. ¿Hacia un nuevo federalismo?, Vitoria-Gasteiz, IVAP.

León, S. y M. Ferrín (2012): ¿Qué factores promueven o dificultan la cooperación intergubernamental en el Estado Autonómico?”, en C. Colino, S. León y M. Ferrín, eds., La práctica de la cooperación intergubernamental en España, 1 edn. Madrid, Centro de Estudios Políticos y Constitucionales, pp. 55-78.

Linz, J. J. (1997): Democracy, Multinationalism, and Federalism, Estudios / Working Papers, Madrid, Centro de Estudios Avanzados en Ciencias Sociales (103).

Lovell, C., A. Mandondo y P. Moriarty (2002): “The Question of Scale in Integrated Natural Resource Management”, Conservation Ecology, 5.

Máiz Suárez, R. (2018): Nacionalismo y federalismo: una aproximación desde la teoría política, 1 edn. Madrid, Siglo XXI de España.

Máiz, R. (2013): "Beyond institutional design: the political culture of federalism (A normative approach)", en VV. AA., The Ways of Federalism in Western Countries and the Horizons of Territorial Autonomy in Spain, Volume I. 1 edn. Springer, pp. 83-102.

Máiz, R. (2005): Estado de las autonomías y federalismo plurinacional. Hacia una España plural, social y federal, 1 edn. Barcelona, Editorial Mediterrània, pp. 161-176.

Máiz, R., P. Beramendi y M. Grau (2002): "La Federalización del estado de las autonomías: evolución y déficit institucionales", en J. Subirats y R. Gallego, eds, Veinte años de Autonomías en España, Madrid, Centro de Investigaciones Sociológicas, pp. 379-424.

Marks, G., L. Hooghe y A. H. Schakel (2008): “Measuring regional authority”, Regional and Federal Studies, 18(2-3), pp. 111121.

Martínez-Fernández, J. (2019): El proceso de evaluación de la Directiva Marco del Agua, Zaragoza, Fundación Nueva Cultura del Agua.

Martínez Herrera, E. (2010): Federalism and ethnic conflict management: rival hypotheses, and attitudinal missing link and comparative evidence. New Directions in Federalism Studies, 1 edn. Abingdon, Routledge, pp. 141-156.

Mondragón, J., A. De La Peña, A. Elizondo, J. L. Mokoroa y P Juaristi (2016): "State and regional administrative coordination in Spain: a case study of Spanish sectoral conferences on environmental, helth and educational policies (2001-2012)", European Policy Analysis, 2(2), pp. 94-117.

Moreno, L. (2009): “La federalización de la España plural”, Revista d'estudis autonòmics i federals, 8, pp. 119-143.

Moreno, L. (2008): La federalización de España: poder político y territorio, 2 edn. Madrid, Siglo XXI de España Editores.

OECD (2011): Water Governance in OECD Countries: A Multi-level Approach, OECD Studies on Water, OECD Publishing. http://dx.doi.org/10.1787/9789264119284-en

Ostrom, E. (2015): El gobierno de los bienes comunes. La evolución de las instituciones de acción colectiva, México D.F., Fondo de Cultura Económica.

Pérez Castaños, S. y J. García Rabadán (2019): "La cultura política federal en España: cambios y consecuencias ante el reto catalán”, REIS: Revista Española de Investigaciones Sociológicas, 167, pp. 37-56.

Pérez Díaz, V., Mezo, J. y Álvarez Miranda, B. (1996): Politica y economía del agua en España: criterios, alternativas y proceso de aprendizaje, Madrid, Círculo de Empresarios.

Romero, J. (2020): "Gobernanza territorial y vieja normalidad política en España. A propósito del modelo de federalismo incompleto y disfuncional", en F. J. Moreno Fuentes y E. Del Pino, eds, las Transformaciones Territoriales y Sociales del Estado en la Edad Digital, Madrid, Centro de Estudios Políticos y Constitucionales, pp. 123-156.

Romero, J. (2005): "El gobierno del territorio en España. Balance de iniciativas de coordinación y cooperación territorial", Boletín de la $A G E, 39$, pp. 59-86.

Sánchez Martínez, M.T., Rodríguez Ferrero, N. y Salas Velasco, M. (2011): "La gestión del agua en España. La unidad de Cuenca", Revista de estudios regionales, 92, pp. 199-222.

Smith, N., 2020. Desarrollo desigual. Naturaleza, capital y la producción del espacio. Madrid, Traficantes de Sueños.

Swyngedouw, E. (2015): "Urbanization and environmental futures: Politicizing urban political ecologies", en Perreault, T., G. Bridge y J. Mccarthy, The Routledge Handbook of Political Ecology, London \& New York, Routledge, pp. 609-619.

Vallès, J. M. (2016): “El Estado de las autonomías: una apuesta fallida”, en C. Colino, ed, Ciencia y Politica: Una Aventura Vital, Valencia, Tirant lo Blanch, pp. 391-418.

VV.AA. (dir. A. Embid Irujo) (1999): Planificación hidrológica y política hidráulica: (el libro blanco del agua), 1 edn. Zaragoza, Civitas, Universidad de Zaragoza, Ministerio de Agricultura, Alimentación y Medio Ambiente.

Wälti, S. (2017): “La gobernanza multinivel del medio ambiente”, en G. Lachapelle y P. Oñate, eds, Federalismo, Devolution y Gobernanza Multinivel, Valencia, Tirant Lo Blach, pp 151-168. 